\title{
S5ynthesis
}

International Scientific Conference of IT and Business-Related Research

\section{LOCATION-BASED APPLICATIONS FOR SMARTPHONES}

\author{
LOKACIJSKI BAZIRANE APLIKACIJE ZA PAMETNE TELEFONE
}

\author{
Vladimir Lazović, Nikola Minić, Milan Tair \\ Singidunum University, 32 Danijelova St., Belgrade, Serbia
}

\begin{abstract}
:
This paper presents an implementation of a location-based service developed for smartphone devices. It also covers a review of the need for location-based services and methods of location acquisition, which include GPS, cell-stations, wireless access point and IP address based Geo-location lookup positioning. The paper covers a review of contemporary technologies for development of software systems based on the location-based services and their use on smartphone devices. The paper presents the implementation of a GIS designed to provide venue information based on the location information of smartphones with Android operating system.
\end{abstract}

\section{Key words:}

Android, Location-Based Service, GPS, Wi-Fi, Smartphone.

\section{INTRODUCTION}

Web application research and development is one of the most prevalent fields in software engineering today. Web applications typically follow a client-server model. The client side is a program called web browser, which is freely available for download by the user. The server side hosts the application code, which is, in its original or modified form, transferred over the web to the client program to be executed. The web application can generate a user-oriented interface, suitable for transferring information to the user. Design and layout changes to the user interface are made by modifying the original application source code hosted on the server.

Earlier content accessible offer the Internet featured statically coded, constant web pages. Concurrent practice is to create dynamically generated web content, personalized, according to the information about the user for whom the content is being generated. This personalization data may include the preferred language and locale, the need for a specialized form of user interface accessibility and other enhancement requirements, as well as the user's location. Modern web applications implement technologies for detecting user preferences in order to provide a more personalized content, prior to a user's request for such adaptation of provided information. Many of these technology implementations still require the user to manually confirm or allow the sending of location information to the website requesting it.

\section{Apstrakt:}

U radu je predstavljena primena usluge bazirane na lokaciji, kreirane za pametne telefone. U njemu se analizira potreba za uslugama baziranim na lokaciji, kao i metode utvrđivanja lokacije, među kojima su GPS, lociranje pomoću baznih stanica, bežičnih mreža i utvrđivanje lokacije pomoću IP adrese. Rad sadrži i pregled savremenih tehnologija za izradu softverskih sistema baziranih na lokaciji i njihovu upotrebu na pametnim telefonima. Predstavljena je implementacija jednog geografskog informacionog sistema dizajniranog da pruži informacije o ugostiteljskim objektima, koje su u blizini pametnih uređaja sa Android operativnim sistemom, bazirane na njihovoj lokaciji.

\section{Ključne reči:}

Android, usluge bazirane na lokaciji, GPS, bežične mreže, pametni telefoni

\section{LOCATION-BASED SERVICES}

Location-based services (LSB) have emerged during the 1970s in the United States of America, when the United States Department of Defence (DOD) started using the Global Positioning System (GPS). The GPS is based on a satellite grid infrastructure. It is used for locating people, and objects. The early GPS implementation was accurate up to three meters (Spiekermann, 2004, p. 10). Originally, GPS was devised as a system that would be utilized by the military. However, in 1980s, the United States Government decided to allow the worldwide use of this system. Ever since, many industries have adopted this technology in order to make improvements of their products and services. Notably, the automobile industry now integrates GPS receivers in vehicles to provide location-based services available to drivers.

An object or a person can be tracked by recording a set of coordinates, which include latitude, longitude, and when calculable, to a degree of certainty, the altitude. The data required in order to provide other connected systems with this information is received by a GPS receiver.

During the 1990s, interest in LBSs and derivative products and services increased, especially with the increase in the use of mobile communication devices. With the increase of the use of LBSs, the need for more precise GPS positioning emerged.

The importance of GPS accuracy for different types of GIS applications is shown in the following table, formed according to (Bellocci, Genovese, Inuaggiato, \& Tucci, 2002). 


\begin{tabular}{|l|l|l|l|}
\hline \multicolumn{1}{|c|}{ Type of application } & \multicolumn{1}{c|}{ Accuracy level } & \multicolumn{1}{c|}{ Type of application } & Accuracy level \\
\hline News & Low & Games & Medium \\
\hline Direction-finding & High & Mobile commerce apps & Medium High \\
\hline Traffic information & Low & Emergency services & High \\
\hline Points of interest & Medium High & Hazardous material transfer & High \\
\hline Yellow pages & Medium Low & Child tracking & Medium High \\
\hline Vehicle navigation & Medium High & Pet tracking & Medium High \\
\hline Personal navigation & High & Electronic Toll Collection & Medium High \\
\hline Fleet management & Low & Remote personnel managing & High \\
\hline Vehicle tracking & Medium High & Location-based advertising & Medium High \\
\hline Asset tracking & High & Location-based payment & Medium Low \\
\hline
\end{tabular}

Table 1: The importance of GPS accuracy for different types of applications of GISs

\subsection{LBS STRUCTURE}

LBS applications aim to provide real-time service, such as route planning, point of interest selection etc. (Meng, Zipf, \& Reichenbacher, 2005). There are two methods for providing services based on geo-location. Either the user requests information, products or services for a geo-location he or she provides, or this information is provided for a geo-location automatically detected by means of a geo-positioning technology. Once the geo-location information is acquired, it is sent to the centre for data processing along with other parameters relevant to the service. This set of information is usually stored in a database for later analysis. Based on the received set of information, the database is queried in such a way as to provide a result set, which includes information on products and services relevant to the data set in the request. This data is geographically tagged, which means that it is weighted as more relevant when the user is closer to its location, than it would be if the user were further away.

Applications that rely on LBSs can be user oriented or device oriented (Spiekermann, 2004, p. 13). User oriented applications are designed to provide information on products and services to users, based on the parameters selected by the user. For instance, these applications can point the user to the nearest computer component store. Device -oriented applications are designed to be self-activated and they usually do not require any input from the user prior to or after activation. These applications are made as integral parts of devices or objects, such as vehicles (for example, a GPS receiver embedded in an automobile). With such an application, users are seldom allowed to manage the device and its functions.

\subsection{LOCATION INFORMATION ACQUISITION METHODS}

The standard method for location acquisition is the use of a GPS receiver. However, there are at least four alternative methods for location determination, which can be used in combination (Business Insider, 2013). These methods are:

- Cell phone towers: When the GPS satellite signal cannot penetrate the surrounding area and reach the receiver, the device may acquire location information from a cell phone base-station. This happens in closed spaces, surrounded by concrete walls. This method is less accurate than GPS positioning;

- Wi-Fi access point: This method is accurate, but required an active Wi-Fi hotspot;

- IP address geolocation: The accuracy of this method depends on the Internet Service Provider (ISP);
- User defined location: When a user registers for a service, he or she may enter the address of residence, which can be used to determine the geolocation.

\subsection{Applications of LBS}

Apart from military use, which originally utilized GPS, many new commercially available solutions and applications based on GPS derived LBSs have emerged (Re \& Ruggieri, 2007). These applications span across almost all major fields.

LBS can be implemented for the following purposes (Buczkowski, 2011): Marketing, Emergency services, Information systems, Navigation, Location-based social networks, Locationbased mobile device games, Electronic Toll Collection, Sports, Identifying points of interest, Multimedia geo-tagging, Tracking and Tourism.

Another implementation of LBSs is in the domain of electronic banking security. This particular implementation requires high accuracy and high security LBSs that can guarantee the determined geo-location coordinates as well as privacy. Most of the time, these implementations require specialized hardware for accurate location providing. Additionally, these systems are seldom modular and their software is often encrypted as well as close-sourced (Ardagna et al., 2009).

\section{TECHNOLOGY REVIEW}

\subsection{MYSQL RDBMS}

MySQL is an open-source relational database management system (RDBMS) for managing databases. It is available under two licenses (Deek \& McHugh, 2007, p. 273). The commercial licence applies when embedding the MySQL server into a commercial solution and the community license applies when connecting to a MySQL server from a commercial or free solution.

An alternative RDBMS, often chosen instead of MySQL has been the PostgreSQL, which is also an open source project. PostgreSQL is available in major distributions of GNU/Linux operating system. However, it is also available for Mac OS X, Windows, etc. MySQL was optimized towards performance at the expense of advance functionalities (Halpin, 2008). Despite limited availability of the advanced SQL constructs, MySQL is a stable and well-documented solution with a wide support for major programming and scripting languages among which are PHP, Java, C, Perl, Python etc.

\subsection{PHP SCRIPT LANGUAGE}


PHP (PHP - Hypertext Pre-processor) is a specialized scripting language, primarily used for the development of dynamic web pages. Its key role is dynamically generating HTML code (Locati et al., 2006), but it has many other applications. By using PHP, it is possible to create an HTML page with dynamically selected and structured content. This way, the PHP code, which had generated the page, cannot be accessed by the user. The user can observe only the output, which is the result of a PHP script execution. The PHP language is similar to that of the $\mathrm{C}$ programming language. Many of the features available in $\mathrm{C}$ are also present in PHP. The most noticeable is its ability to be used as a procedural language. However, PHP can also be used in an object-oriented manner. PHP is available on most major platforms, such as GNU/Linux, Mac OS X and Windows.

\subsection{THE JQUERY JAVASCRIPT LIBRARY}

JavaScript is an object-oriented programming language designed for the development of dynamic websites. Millions of websites use JavaScript to improve user interaction, add functionality, validate form inputs, detect browsers, enable asynchronous communication between a web page and the server, etc. It was developed in 1995 by Netscape to be used as a clientside Internet programming language. This means that JavaScript code is evaluated and executed on the user's computer, within the browser process. This allows for lower web server load, since much of the processing is passed to the client's computer. In addition, web applications can be made to respond instantly to user-triggered events and thus achieve greater efficiency.

\subsection{ANDROID OPERATING SYSTEM}

The Android operating system is the most widely used cellphone operating system in the World. Android is based on the Linux kernel. It was designed to provide better security, power consumption optimisation and an integrated support for touch screens. The Android operating system was optimized to run on devices such as cell phones, tablet computers, laptop computers, smart book computers, electronic book readers, television sets, automobiles and wristwatches (Elenkov, 2014).

Several Android operating system versions have been released since the introduction of the Android 1.0. The 5.1 version, called Lollipop is currently the newest available version of Android. Other Android versions that exist are used around the World in parallel. Those are 4.2 called Jelly Bean, 4.3 also called Jelly Bean and 4.4 named KitKat (Yaghmour, 2013).

\subsection{GEOGRAPHICAL INFORMATION SYSTEMS}

Geographical Information Systems (GIS) are systems comprised of high performance computer hardware, specialized software and data. GIS employ many trained professional personnel, required for system maintenance and administration. All parts of the system are connected through computer networks. All data are stored in the extensive and sometimes redundant database for efficient storage, manipulation, analysis, structuring, diffusion and reporting of geo-location data.

Modern GIS concepts imply that the world is made up of objects. Spatial objects are objects that have a spatial reference. They have a unique identification number and meta information that describe their geometric, thematic, radiometric and temporal attributes.

Every GIS consists of three components (Sharma, Prasad,
\& Binda):

- Computer Hardware and Software;

- Spatial data; and

- Trained personnel.

Computer hardware consists of computers and computer networks that run and connect the GIS and its components. The software can run on a variety of hardware, from server architectures to personal computers. GIS software is a specialized kind of software that provides functions and utilities required for storing geographical information. It also provides tools for querying, analysing and generating of reports, images, mapped data, tables etc. All GIS software packages rely on database management systems (DBMS), which allows for efficient data analysis and querying.

Objects' special information is among the most important and usually the most expensive component of any GIS. Geographical data structures include special coordinates and bounds, temporal coordinates and bounds, thematic tags and other meta information. This data is usually stored in the GIS database after being converted from analogue sources through a process called digitalisation (Davison et al., 2005). This process includes digital encoding of geographical characteristics, such as buildings, roads, regions and country borders.

The real benefit of these systems becomes evident when analysing the way people use them. Over the last decade, computing units have become much smaller and lighter, yet they have also become more accessible to people. Having this in mind, many new GISs have been created by companies and organizations that offer information, products and services through these systems, which are easily accessible through handheld, portable devices. The number of such systems is growing. People use these systems in a wide variety of fields. Most of the time, these systems allow people to better organize their activities and more efficiently find relevant information, especially if the geographical proximity of the object of interest covered by that information is near to their current location.

\subsection{WEBGIS}

WebGIS is a distributed information system, which is comprised of a GIS application server and client applications. Client applications are mostly in the form of web applications, desktop applications or mobile phone applications (see Fig. 1). The server is accessible on the web via a URI.

All client side applications rely on the HTTP specification to send requests to the server and receive responses from the server. The server can provide the response in a number of widely supported data formats. Responses can be formatted in the form of an HTML page, which can be viewed using a web browser. However, other machine-friendly formats are also supported, such as a binary file with a predefined binary structure, XML (Extensible Markup Language) or the JSON (JavaScript Object Notation) format structured information.

There are two kinds of WebGIS application architectures: the client side and the server-side application architecture.

The client side application architecture aims to transfer most of the processing to the client, instead of having the server perform analysis and generating of data presentation. Some applications developed with this architectural concept require the user to install special software on the client's computer in order to attain access to data. This software usually comes in a form of a web browser extension or plug-in. In other cases, a complete stand-alone software suite installation is required. Nevertheless, both scenarios require the user to install additional software in 
order to utilize the WebGIS system designed in the client side application architecture concept.

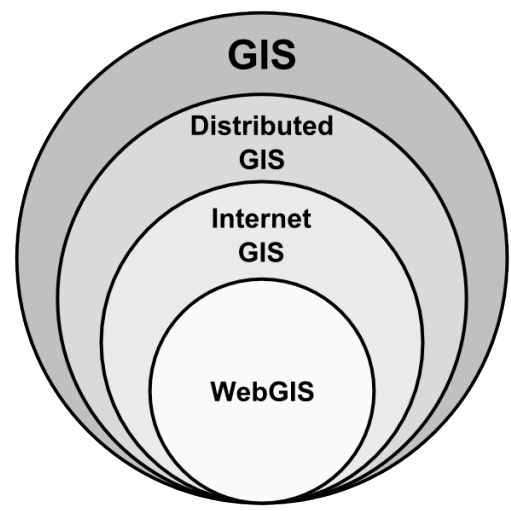

Figure 1. Terminology relationships between different subtypes of GIS.

Source: Jovanović et al. (2012, p. 154)

The server side application architecture aims to have the user send a simple request to the server and have the server execute all necessary tasks. These tasks include data analysis, filtering, sorting and formatting before sending the results back to the client through the standard HTTP protocol response. The response contains a complete web page formatted in a manner understandable by a human. The advantage of this architecture concept is that GIS data is centralised on the server, which simplifies development, modifying, augmenting and maintenance.

\section{THE GIS API AND THE SMARTPHONE APPLICATION}

Our implementation of a GIS is built on an open source platform by using contemporary web development technologies. The base platform is a GNU/Linux operating system. We have used a shared hosting solution for the actual platform, whose resources are divided between a number hosting users. The server is located in a datacentre company based in Germany, which also provides a fast Internet connection to the central nodes in Serbia.

\subsection{THE GIS DATABASE}

The database is tasked with storing and processing data. Like other parts of the system, we have opted to use open source software. The preferred Relational DBMS (RDBMS) chosen was the MySQL RDBMS. As explained in the TECHNOLOGY REVIEW section, MySQL is a relational model DBMS, which is open source and has been optimized for performance. Another key advantage is its dual license model, which allows for free use of the MySQL database server in conjunction with other, possibly commercial solutions. Even though MySQL is supported by the majority of programming languages used today, including $\mathrm{C} / \mathrm{C}++, \mathrm{C}$, Java, Python, Perl, etc., its native support by PHP was one of the main reasons for its selection. The database server in our implementation was installed on the same web server, which hosts the GIS API software. It is possible to install the database server on a dedicated server, or a cluster of servers.

\subsection{THE GIS API SOFTWARE}

The most important part of our implementation of GIS is the GIS API. Application Program Interfaces (APIs) provide programmers with a way to develop applications without having to access the database directly. Also, the use of APIs guarantees that all programs using a common API will have similar interfaces (Chang \& Hwang, 2007). The GIS API functions as an interface between the database and the mobile device applications. The GIS API is a web-based service written in PHP scripting language, targeting the 5.5 version of the language. As one of the most popular web development languages, with a total market share of over 75\% (Olsson, 2013), PHP was chosen as a preferred language to write the API software. An advantage of PHP is that it can be embedded directly into HTML within the same file (Dahl, Banerjee, \& Spalti, 2006), but with a file extension dedicated to PHP scripts. The API uses new features, introduced in the latest versions of PHP, and is written in an object-oriented paradigm.

\subsection{THE SMARTPHONE APPLICATION}

The application ${ }^{1}$ was developed for smartphone devices with the Android operating system. The communication between the smartphone application and API is achieved through the HTTP protocol's standard HTTP request calls. The API was instructed to return results in XML format.

The smartphone application required a set of special permissions to be granted during installation. These permissions ensure that it can use the GPS location provider or the crude location provider. Both of these location providers are integral parts of the Android operating systems (Software Development Kit) SDK (Meier, 2009). These two providers allow the application to acquire the current or the last known approximate geo-location of the device. If a GPS signal is available, the provider will use it to determine the coordinates and if not, it will use the cell phone base-station signal. When the application is started, it acquires the current location and checks for an active Internet connection, which is necessary for the application to work. After an Internet connection is made, the application will request the API to send a list of venues with basic information embedded in the response. This information includes a set of coordinates, which will be used to display a venue list sorted distance from the device, in an ascending order.

\section{THE CLIENT-SERVER COMMUNICATION}

The communication between the smartphone application and the server hosted GIS API is accompanied by sending data in a prearranged format. The smartphone application sends a standardized HTTP application layer protocol GET method request to the GIS API, which takes arguments and instructions. The GIS API than connect to the GIS database and queries the required data, based on the supplied set of arguments. These arguments, among other data, convey the geo-location coordinates of the user. Based on these instructions and arguments, the GIS API compiles a response in form of an XML structured set of elements. These XML elements contain information needed by the smartphone application to display a list of venues, which the user can than further review in more detail. The GIS API also provides such detailed information sets when such data are requested through future API calls made by the smartphone application.

1 Our smartphone application DEMO version is available at http:// goo.gl/OeGkAl 


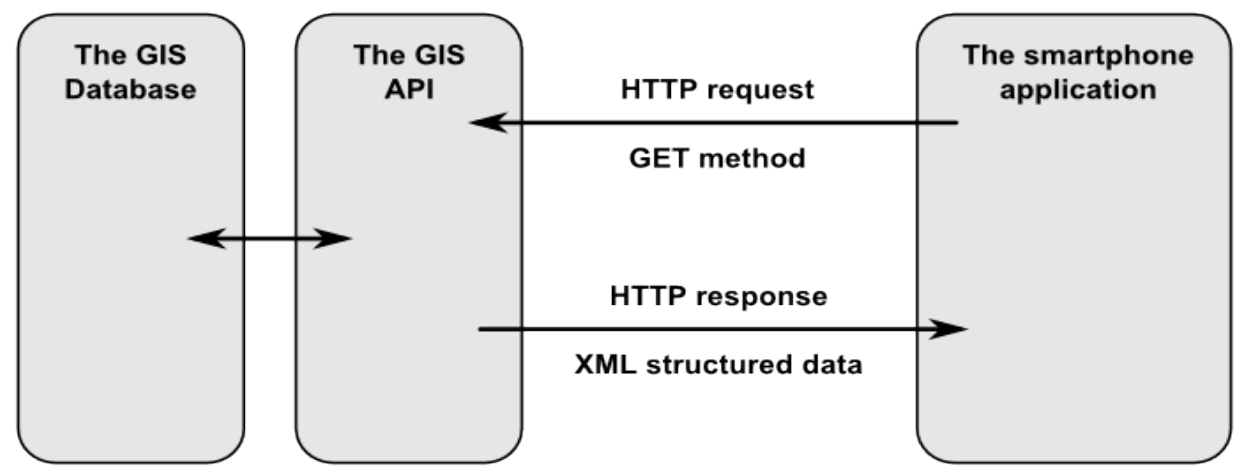

Figure 2. A generalized communication diagram between fragments of our GIS implementation

The communication diagram shown in Fig. 2 illustrates a generalized concept of communication implemented between our GIS API and the smartphone application. The diagram shows the data channel direction as well as the type of data being exchanged between the GIS API and the application. The server side of the diagram depicts an abstraction of a database, with a bi-directional connection between the database and the GIS API software.

\section{CONCLUSION}

This paper covers a review of Location-Based Services, GPS technology and Web Development Technologies. In addition, we have presented a review of an own implementation of a Geographical Information System, specially designed to be accessed from an application installed on a smartphone device running an Android operating system. The GIS is comprised of a relational model database, an API and a user application for smartphone devices. The communication between the application and the API is accomplished through standard HTTP application layer protocol data transfer. Requests to the API are made through the HTTP GET method and API responses are structured in the XML format. The GIS provides information on the location of venues, such as restaurants and cafés near the user. Future work on this project will include acquisition of more venue location data, contact information, promotional content and special offers to users of the smartphone application and commercialisation of the service.

\section{REFERENCES}

Ardagna, C.A., Cremonini, M., Vimercati, S.D., \& Samarati, P. (2009). Access Control in Location-Based Services. Privacy in Location-Based Applications, 5599, 106-126.

Bellocci, V., Genovese, S., Inuaggiato, D., \& Tucci, M. (2002). Mobile Location-Aware Services: 2002 Market Perspective. Ericsson, Divison Service Architecture and Interactive Solutions.

Buczkowski, A. (2011). Location-Based Services - Applications. Retrieved march 20,2014, from http://geoawesomeness.com/ knowledge-base/location-based-services/location-basedservices-applications/

Business Insider. (2013, October). Infographic: How Location Data Is Collected To Power The New Generation Of Mobile Marketing Campaigns. Retrieved March 2014, from Business Insider: http://businessinsider.com/mobile-location-basedservices-and-location-data-2013-8
Chang, T.-K., \& Hwang, G.-H. (2007). The design and implementation of an application program interface for securing XML documents. Journal of Systems and Software, 80(8), $1362-1374$

Dahl, M., Banerjee, K., \& Spalti, M. (2006). Digital Libraries: Integrating Content and Systems. Amsterdam: Elsevier.

Davison, E.R., Harris, R.W., Qureshi, S., Vogel, D.R., \& Vreede, G.-J. D. (2005). Information Systems in Developing Countries: Theory and Practice. Kowloon Tong: City University of HK Press.

Deek, F. P., \& McHugh, J. A. (2007). Open Source: Technology and Policy. Cambridge: Cambridge University Press.

Elenkov, N. (2014). Android Security Internals: An In-Depth Guide to Android's Security Architecture. San Francisco: No Starch Press.

Halpin, T. (2008). Selected Readings on Database Technologies and Applications. Hershey, PA, United States of America: IGI Global.

Jovanović, V., Đurđev, B., Srdić, Z., \& Stankov, U. (2012). Geografski informacioni sistemi. Belgrade and Novi Sad: Singidunum University \& University of Novi Sad.

Locati, M., Meletti, C., Rovida, A., Rubbia, G., Ercolani, E., \& Meroni, F. (2006). A WebGIS tool for the dissemination of earthquake data. European Geosciences Union - General Assembly 2006. Vienna: European Geosciences Union.

Meier, R. (2009). Professional Android Application Development. Hoboken: John Wiley \& Sons.

Meng, L., Zipf, A., \& Reichenbacher, T. (2005). Map-Based Mobile Services: Theories, Methods and Implementations. Berlin: Springer Science \& Business Media.

Olsson, M. (2013). PHP Quick Scripting Reference. New York City: Apress, Springer Science+Business Media.

Re, E., \& Ruggieri, M. (2007). Satellite Communications and Navigation Systems. Berlin: Springer Science \& Business Media.

Sharma, H. S., Prasad, R., \& Binda, P. (2006). Mathematical Modeling In Geographical Information System (GIS) \& GPS An Overview. New Delhi, India: Concept Publishing Company. Retrieved October 12, 2014

Spiekermann, S. (2004). General Aspects of Location-Based Services. In J. Schiller, \& A. Voisard, Location-Based Services (pp. 9-26). Elsevier.

Yaghmour, K. (2013). Embedded Android: Porting, Extending, and Customizing. Sebastopol, CA, USA: O’Reilly Media, Inc. 